\title{
Gene-environment interplay and the importance of self-control in predicting polydrug use and substance-related problems ${ }^{\text {ts }}$
}

\author{
Michael G. Vaughn ${ }^{\text {a,*}}{ }$, Kevin M. Beaver ${ }^{\mathrm{b}}$, Matt DeLisi ${ }^{\mathrm{c}}$, Brian E. Perron ${ }^{\mathrm{d}}$, Lisa Schelbe ${ }^{\mathrm{e}}$ \\ a Saint Louis University, St. Louis, MO, United States \\ b Florida State University, Tallahassee, FL, United States \\ c Iowa State University, Ames, IA, United States \\ d University of Michigan, Ann Arbor, MI, United States \\ e University of Pittsburgh, Pittsburgh, PA, United States
}

\section{A R T I C L E I N F O}

\section{Keywords:}

Etiology

Genes and environment

Biosocial

Adolescent drug use

Drug-related problems

\begin{abstract}
A B S T R A C T
Using the National Longitudinal Study of Adolescent Health (Add Health), the current study applies a general biosocial theoretical model to polydrug use and associated substance-related problems. Along with measures of molecular genetic polymorphisms, neurocognitive skills, self-control, and environmental pathogens a recursive path modeling strategy was used to empirically examine the relations between these biosocial measures and polydrug use, alcohol, and drug-related problems in a subsample of 1136 adolescent males (Mean age $=21.96$, $\mathrm{SD}=1.73$ ). Results supported the main predictions of the biosocial model finding significant path coefficients across key constructs. In particular, the role of poor self-regulation was found to be sturdy across path models.
\end{abstract}

(c) 2008 Published by Elsevier Ltd.

\section{Introduction}

The use and abuse of alcohol and other drugs and their resulting consequences involve processes influenced by genetic, physiological, psychological and environmental factors. The justification for employing multiple disciplinary constructs in understanding substance abuse and substance-related problems is that these behavioral phenotypes are clearly multidimensional in nature and will necessarily involve a host of disciplines (e.g., genetics, neuroscience, endocrinology, psychology, sociology). The current study is unique because few studies have examined the biosocial interplay in relation to polydrug use and substancerelated problems that have combined genes, proximal environmental mechanisms, and self-control. Two main propositions are tested in the present study: 1) Causal pathways to polysubstance use and substance-related problems will involve associations among genes, proximal environmental pathogens, and cognitive impairments and 2) The effects of self-control will be stable across polysubstance use, alcohol-related problem behavior and drug-related problem behavior.

\footnotetext{
is This research uses data from Add Health, a program project designed by J. Richard Udry, Peter S. Bearman, and Kathleen Mullan Harris, and funded by a grant P01-HD31921 from the Eunice Kennedy Shriver National Institute of Child Health and Human Development, with cooperative funding from 17 other agencies. Special acknowledgment is due to Ronald R. Rindfuss and Barbara Entwisle for assistance in the original design. Persons interested in obtaining data files from Add Health should contact Add Health, Carolina Population Center, 123 W. Franklin Street, Chapel Hill, NC 27516-2524 (addhealth@unc.edu). No direct support was received from grant P01-HD31921 for this analysis.

* Corresponding author. Saint Louis University, Tegeler Hall, 3550 Lindell Boulevard, St. Louis, MO 63103, United States. Tel.: +1 3149772718.

E-mail address: mvaughn9@slu.edu (M.G. Vaughn).
} 


\section{Methods}

\subsection{Data}

Data for this study come from the National Longitudinal Study of Adolescent Health (Add Health) DNA subsample. Information regarding the design and procedures has been published elsewhere (e.g., Beaver, DeLisi, Wright, \& Vaughn, 2008). Following prior researchers analyzing the genetic subsample of the Add Health (Haberstick et al., 2005), we removed one twin from each MZ twin pair to provide conservative parameter estimates. The analyses were confined to 1136 male respondents (Mean age $=21.96$, $\mathrm{SD}=1.73)$.

\subsection{Measures}

\subsubsection{Dopamine transporter gene (DAT1) and dopamine D2 receptor gene (DRD2)}

The dopamine transporter gene (DAT1) is responsible for coding for the production of the dopamine transporter protein. DAT1 has a 40 base pair variable number of tandem repeats in the $3^{\prime}$ untranslated region of the gene (SLC6A3). Following prior researchers working with the Add Health data (Hopfer et al., 2005), participants who had alleles other than the 9R allele or the 10R allele were removed from the sample. The DAT1 variable was coded to indicate the number of 10R alleles that each person possesses.

The dopamine D2 receptor gene (DRD2) codes for the production of the D2 receptor, and has a polymorphic TaqI restriction endonuclease site that is 2500 base pairs downstream from the gene's coding region (Grandy et al., 1989). The minor TaqIA (A-1 allele) has a point mutation, where $\mathrm{C} \rightarrow \mathrm{T}$ (TCGA to TTGA) that eliminates the TaqI site. The DRD2 variable indexes the number of A1 alleles that each person possesses. Hardy-Weinberg equilibrium was fulfilled for DAT1 and DRD2 in the Add Health sample.

\subsubsection{Neurocognitive skills}

During wave 1 interviews, respondents completed the Peabody Picture Vocabulary Test (PPVT-R), which is a standardized test designed to assess verbal ability and receptive vocabulary. Higher scores on the PPVT indicate more verbal abilities and less neurocognitive deficits (range $=38-133$ ).

\subsubsection{Deviant peer affiliations}

The Add Health data contain three different items that measure contact with and exposure to deviant peers. The three items were summed together to form the wave 1 delinquent peers scale, where higher scores reflect more delinquent peers $(\alpha=.74)$.

\subsubsection{Maternal withdrawal}

The Add Health study contains five different items asked to the respondent at wave 1 that measure maternal withdrawal. The five items were summed together and the scale was coded such that higher values indicate more maternal withdrawal $(\alpha=.79)$.

\subsubsection{Attention deficit hyperactivity disorder symptoms}

At wave 3, eighteen different questions were used to measure the degree to which each respondent was hyperactive between the ages of five and twelve years old. The items were then summed to form the retrospective ADHD scale ( $\alpha=.90)$.

\subsubsection{Self-control}

During wave 3 interviews, respondents were asked nine questions designed to capture individual variation in levels of selfcontrol. Responses to these items were then summed together to form the wave 3 low self-control scale, where higher scores reflected lower levels of self-control $(\alpha=.85)$

\subsection{Outcome variables}

\subsubsection{Polydrug use}

This outcome variable was operationalized as the use $(0=$ no, $1=y e s)$ of six possible substances including alcohol, marijuana, cocaine, crystal methamphetamine, any injectable drug, and other drugs (e.g., LSD, ecstasy) $(M=1.30, S D=1.10$, range $=0-6)$.

\subsubsection{Alcohol and drug-related problems}

Respondents were queried on alcohol problems based on the past twelve month usage. This scale consisted of eight items $(M=3.05, \mathrm{SD}=4.26$, range $=0-24)$ and possessed adequate reliability $(\alpha=.78)$. As with alcohol-related problems, drug-related problems were based on the past twelve month usage. This scale $(\alpha=.72)$ was comprised of six items tapping problems related to drug use $(M=.59, \mathrm{SD}=1.94$, range $=0-18)$.

\subsection{Analytic strategy}

A series of structural equation models were calculated employing maximum likelihood estimation specifically examining the interrelationships among the aforementioned genotypic, neurodevelopmental deficits, proximal environmental pathogens and 


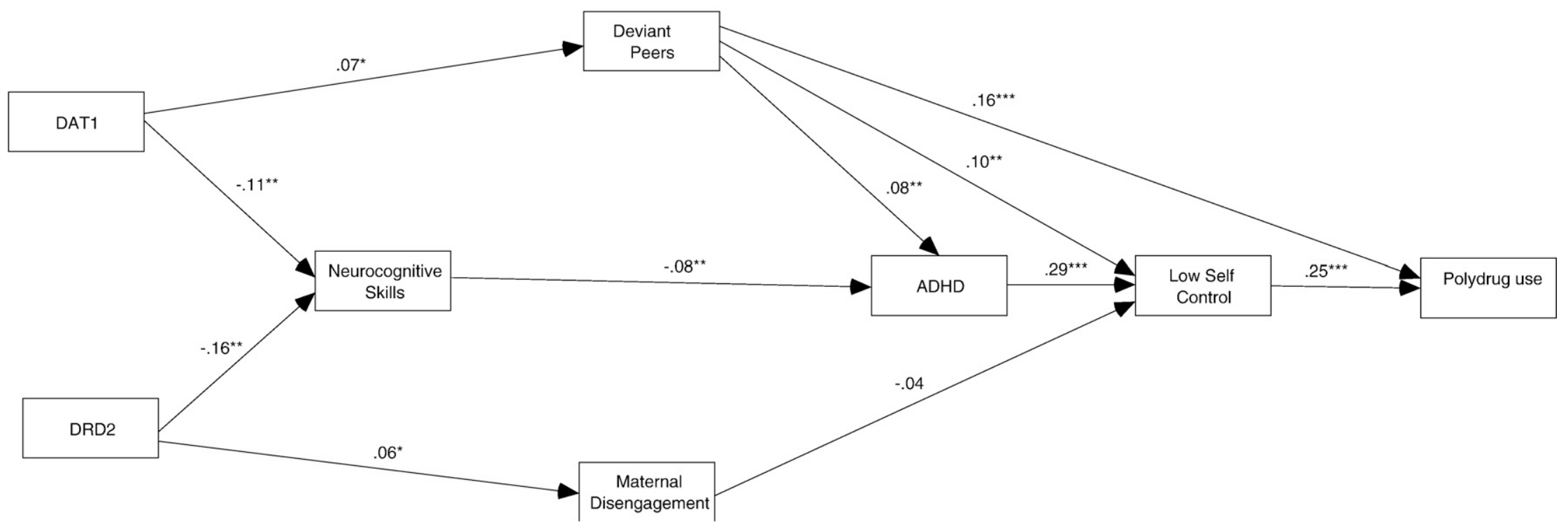

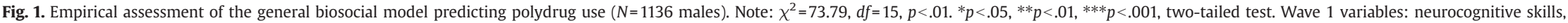
deviant peers, maternal disengagement. Wave 3 variables: genes, ADHD, self-control, polydrug use. 


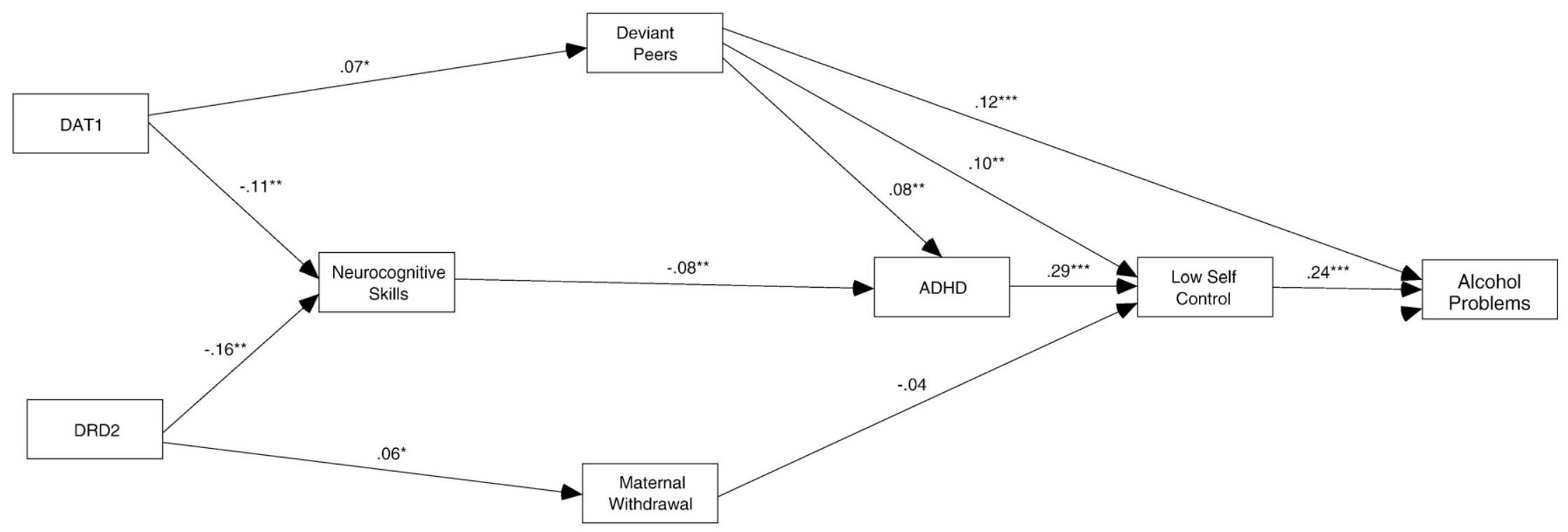

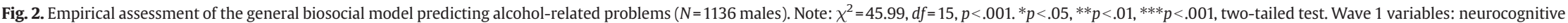
skills, deviant peers, maternal disengagement. Wave 3 variables: genes, ADHD, self-control, polydrug use. 
self-control measures. Our models represent a theory test rather than a series of iterations and are thus presented as untrimmed. Structural equation models were calculated for three different dependent measures: the polydrug use scale, the alcohol problems scale, and the drug problems scale.

\section{Results}

Findings begin with the polydrug use scale $\mathrm{CFI}=.843$, RMSEA $=.059 ; \chi^{2}=73.79, d f=15, p<.01$ ) as the dependent variable (see Fig. 1). As hypothesized, the measures tapping a vulnerable genotype (i.e., DAT1 and DRD2) were predictive of neurocognitive skills, meaning that respondents with more DAT1 risk alleles (Beta $=-.11, p<.05$ ) or with more DRD2 risk alleles (Beta $=-.16, p<.05)$ had diminished neurocognitive skills. Measures of a vulnerable genotype also had significant relationships with environmental pathogens; DAT1 was positively associated with deviant peers (Beta=.07, $p<.05$ ) and DRD2 was positively associated with maternal withdrawal (Beta $=.06, p<.05$ ). As can be seen, deviant peers had a statistically significant and positive effect on ADHD $($ Beta $=.08, p<.05)$, on low self-control (Beta $=.10, p<.01$ ), and on delinquency (Beta $=.16, p<.001$ ). Maternal withdrawal scale was not significantly related to self-control or polydrug use. ADHD has a significant positive effect on low self-control (Beta=.29, $p<.001$ ) and low self-control has a significant positive effect on polydrug use (Beta $=.25, p<.001$ ).

Our next two models examined the effects of biosocial interplay on alcohol (see Fig. 2$)\left(\mathrm{CFI}=.903, \mathrm{RMSEA}=.043 ; \chi^{2}=45.99\right.$, $d f=15, p<.001)$ and drug-related problems (not shown) (CFI=.971, RMSEA $\left.=.021 ; \chi^{2}=22.70, d f=15, p=.091\right)$. The results of these two models once again supported the main predictions of the general biosocial model. The effects of deviant peers on alcohol problems (Beta $=.12, p<.001$ ) were slightly stronger than on the drug problems measure (Beta $=.07, p<.01)$. Low self-control was positively correlated with alcohol problems (Beta $=.24, p<.001)$ and drug problems $($ Beta $=.29, p<.001)$.

\section{Discussion}

The overall aim of the present study was to provide a preliminary test of a general biosocial liability model in predicting polydrug use and substance-related problems. Study findings showed respondents with more DAT1 risk alleles (i.e., 10R alleles), or with more DRD2 risk alleles (i.e., A-1 alleles) had reduced neurocognitive skills. Consistent with the general contours of the biosocial liability model, DAT1 was positively associated with delinquent peers and DRD2 was positively associated with maternal withdrawal. The findings suggest exposure to environmental pathogens by males with vulnerable genotypes may begin to differentially set an individual toward the use of multiple drug and substance-related problems-a finding consistent with the overall model. Further, deviant peer affiliations had a positive effect on measures of ADHD, low self-control, and all three outcome variables. Exposure to deviant peers was associated with increased ADHD symptoms, reduced levels of behavior control and polydrug use and problems stemming from the use of alcohol and drugs. ADHD symptoms had a significant positive effect on low self-control and in turn low self-control had consistently moderately strong effects on the outcome measures. Thus, our second proposition that the effects of self-control will be sturdy across polysubstance abuse, alcohol-related problem behavior and drugrelated problem behavior was supported.

Consistent with prior research on self-regulatory processes such as neurodisinhibition (Tarter et al., 2003) and impulsivity (Semple, Zians, Grant, \& Patterson, 2005), poor self-control was predictive of drug use and substance-related problem behaviors presumably due to deficits in inhibitory control. Results need to be interpreted within a context of several limitations. First, the study sample was comprised of males only. Thus, a larger study with a higher base rate of problem behavior and polydrug use is necessary to test the validity of the biosocial liability model in females. Second, available measures of neurocognitive skills and selfregulation were not extensive and therefore more precise delineation of neuropsychological functioning was limited.

\section{References}

Beaver, K. M., DeLisi, M., Wright, J. P., \& Vaughn, M. G. (2008). Desistance from delinquency: The marriage effect revisited and extended. Social Science Research, 37, 736-752.

Grandy, D. K., Litt, M., Allen, L., Bunzow, J. R., Marchionni, M., Makam, J. R., et al. (1989). The human dopamine D2 receptor gene is located in chromosome 11 at q2223 and identifies a Taq I polymorphism. American Journal of Human Genetics, 45, 778-785.

Haberstick, B. C., Lessem, J. M., Hopfer, C. J., Smolen, A., Ehringer, M. A., Timberlake, A., et al. (2005). Monoamine oxidase A (MAOA) and antisocial behaviors in the presence of childhood and adolescent maltreatment. American Journal of Medical Genetics, 135B, 59-64.

Hopfer, C. J., Timberlake, D., Haberstick, B. C., Lessem, J. M., Ehringer, M. A., Smolen, J. M., et al. (2005). Genetic influences on quantity of alcohol consumed by adolescents and young adults. Drug and Alcohol Dependence, 78, 187-193.

Semple, S. J., Zians, J., Grant, I., \& Patterson, T. L. (2005). Impulsivity and methamphetamine use. Journal of Substance Abuse Treatment, $29,85-93$.

Tarter, R. E., Kirischi, L., Mezzich, A., Cornelius, J., Pajer, K., Vanyukov, J. M., et al. (2003). Neurobehavior disinhibition in childhood predicts early age onset substance use disorder. American Journal of Psychiatry, 160, 1078-1085. 